\title{
Global stability in discrete models of nonautonomous Lotka-Volterra type
}

\author{
Yoshiaki MUROYA $^{1}$ \\ (Received February 14, 2002; Revised October 17, 2002)
}

Abstract. In this paper, we establish sufficient conditions for the global asymptotic stability of the following discrete models of nonautonomous Lotka-Volterra type:

$$
\left\{\begin{aligned}
N_{i}(p+1)=N_{i}(p) \exp \left\{c_{i}(p)-a_{i}(p) N_{i}(p)-\sum_{j=1}^{n} \sum_{l=0}^{m} a_{i j}^{l}(p) N_{j}\left(p-k_{l}\right)\right\} \\
1 \leq i \leq n, \text { for } p=0,1,2, \ldots, \\
N_{i}(p)=N_{i p} \geq 0, \text { for } p \leq 0, \text { and } N_{i 0}>0,1 \leq i \leq n,
\end{aligned}\right.
$$

where each $c_{i}(p), a_{i}(p)$ and $a_{i j}^{l}(p)$ are bounded for $p \geq 0$ and

$$
\left\{\begin{array}{l}
\inf _{p \geq 0} a_{i}(p)>0, \quad a_{i i}^{0}(p) \equiv 0, \quad a_{i j}^{l}(p) \geq 0,1 \leq i \leq j \leq n, 0 \leq l \leq m, \\
k_{0}=0, \text { integers } k_{l} \geq 0,1 \leq l \leq m .
\end{array}\right.
$$

We establish a condition for the permanence of system and applying the former work (2002, J. Math. Anal. Appl. 273 492-511) to this system, we improve a known condition for the global asymptotic stability of system.

Key words: permanence, global asymptotic stability, discrete model of nonautonomous Lotka-Volterra type.

\section{Introduction}

Consider the following discrete system of nonautonomous LotkaVolterra type:

$$
\left\{\begin{array}{r}
N_{i}(p+1)=N_{i}(p) \exp \left\{c_{i}(p)-a_{i}(p) N_{i}(p)-\sum_{j=1}^{n} \sum_{l=0}^{m} a_{i j}^{l}(p) N_{j}\left(p-k_{l}\right)\right\} \\
1 \leq i \leq n, \text { for } p=0,1,2, \ldots \\
-N_{i}(p)=N_{i p} \geq 0, \text { for } p \leq 0, \text { and } N_{i 0}>0,1 \leq i \leq n
\end{array}\right.
$$

2000 Mathematics Subject Classification : 34D23.

${ }^{1}$ Research partially supported by Waseda University Grant for Special Research Projects 2001A-571. 
where we assume that each $c_{i}(p), a_{i}(p)$ and $a_{i j}^{l}(p)$ are bounded for $p \geq 0$ and

$$
\left\{\begin{aligned}
\inf _{p \geq 0} a_{i}(p)>0, \quad a_{i i}^{0}(p) & \equiv 0, \quad a_{i j}^{l}(p) \geq 0 \\
& 1 \leq i \leq j \leq n, 0 \leq l \leq m \\
k_{0}=0, & \text { integers } k_{l} \geq 0,1 \leq l \leq m
\end{aligned}\right.
$$

For the system (1.1)-(1.2), there are several literatures in autonomous cases. In the case of a prey-predator system for $n=2$ and $m=0$, or the two species are competitive, then Hofbauer, Hutson and Jansen [7] offered a theorem that the existence of positive equilibrium in the system guarantees its permanence. But Lu and Wang [8] show that if the system is cooperative, it can not be permanent in any case. For no delay case $m=0, \mathrm{Lu}$ and Wang [8] also give sufficient conditions for permarence. In the case $n=2$ and any $m \geq 0$, Saito, Ma and Hara [16] and Saito, Hara and $\mathrm{Ma}$ [15] generalized them and established the necessary and sufficient conditions for permanence (see also Muroya [11]).

On the other hand, Wang and Lu [19] and Wang et al. [20] fined further conditions in the case of prey-predator and competitive system for $n=2$ and $m \geq 0$, to ensure that the discrete system is globally asymptotically stable.

Recently, for the cases $n \geq 2$ and $m \geq 0$, applying the techniques offered by Ahmad and Lazer [2] and Muroya [12], Muroya [13] established sufficient conditions for the persistence and global asymptotic stability of the system (1.1) $-(1.2)$.

In this paper, using results in Muroya [13] to the discrete system (1.1)(1.2) of nonautonomous Lotka-Volterra type, we establish a condition for the permanence of system (cf. Wang et al. [20]). For the global asymptotic stability of the system (1.1)-(1.2), we apply also Muroya's results in [13]. In particular, for the autonomous case of $n=1$, we improve the result offered by Muroya [9] for conditions of the global asymptotic stability. This is other type condition than those established by So and $\mathrm{Yu}$ [17] and Muroya [10] (see Remark 2.4).

For a given sequence $\{g(p)\}_{p=0}^{\infty}$, we set

$$
\left\{\begin{array}{c}
g_{M}=\sup \{g(p) \mid p=0,1,2, \ldots\} \\
g_{L}=\inf \{g(p) \mid p=0,1,2, \ldots\}
\end{array}\right.
$$


and put

$$
\left\{\begin{array}{l}
a_{i j L}^{l}=a_{i j L}^{l-}+a_{i j L}^{l+}, \quad a_{i j L}^{l-} \leq 0 \leq a_{i j L}^{l+} \\
a_{i j M}^{l}=a_{i j M}^{l-}+a_{i j M}^{l+}, \quad a_{i j M}^{l-} \leq 0 \leq a_{i j M}^{l+} \\
b_{i j L}^{-}=\sum_{l=0}^{m} a_{i j L}^{l-}, \quad b_{i j M}^{-}=\sum_{l=0}^{m} a_{i j M}^{l-}, \quad b_{i j M}^{+}=\sum_{l=0}^{m} a_{i j M}^{l+} \\
1 \leq i, j \leq n \\
A_{L}=\operatorname{diag}\left(a_{1 L}, a_{2 L}, \ldots, a_{n L}\right), B_{L}^{-}=\left[b_{i j L}^{-}\right], B_{M}^{+}=\left[b_{i j M}^{+}\right] \text {and } \\
D_{M}^{+}=\operatorname{diag}\left(b_{11 M}^{+}, b_{22 M}^{+}, \ldots, b_{n n M}^{+}\right) \text {are } n \times n \text { matrices, and } \\
c_{L}=\left[c_{i L}\right] \text { and } c_{M}=\left[c_{i M}\right] \text { are } n \text {-dimensional vectors. }
\end{array}\right.
$$

For the system (1.1)-(1.2), assume the following condition:

$$
\left(A_{L}+B_{L}^{-}\right)^{-1} c_{M}>0 \text {. }
$$

For (1.4), put

$$
\left\{\begin{array}{l}
\tilde{c}_{i M}=c_{i M}-\sum_{j=1}^{i-1} b_{i j L}^{-} \bar{N}_{j}, \\
\bar{N}_{i}= \begin{cases}\tilde{c}_{i M} / a_{i L}, & \tilde{c}_{i M} \leq 1, \\
e^{\tilde{c}_{i M}-1} / a_{i L}, & \tilde{c}_{i M}>1,\end{cases} \\
\tilde{a}_{i L}=\min \left(a_{i L}, \frac{2}{\bar{N}_{i}}-a_{i M}\right)>0,1 \leq i \leq n, \\
\tilde{A}_{L}=\operatorname{diag}\left(\tilde{a}_{1 L}, \tilde{a}_{2 L}, \ldots, \tilde{a}_{n L}\right), \\
\text { and } \bar{N}=\left[\bar{N}_{i}\right] .
\end{array}\right.
$$

We refer that the system (1.1)-(1.2) is permanent, if there are positive constants $\delta$ and $\gamma$ such that

$$
\begin{array}{r}
0<\delta \leq \liminf _{p \geq 0} N_{i}(p) \leq \limsup _{p \geq 0} N_{i}(p) \leq \gamma<+\infty \\
1 \leq i \leq n
\end{array}
$$

We shall establish the following results to the system (1.1)-(1.2).

Theorem 1.1 Assume the conditions (1.5) and

$$
c_{i L}-\sum_{j=1}^{i-1} b_{i j M}^{-} \underline{N}_{j}-\sum_{j \neq i} b_{i j M}^{+} \bar{N}_{j}>0, \quad 1 \leq i \leq n
$$


where

$$
\left\{\begin{aligned}
\tilde{a}_{i M}=a_{i M} & +\sum_{l=1}^{m} a_{i i M}^{l} \\
& \times \exp \left\{-k_{l}\left(c_{i L}-a_{i M} \bar{N}_{i}-\sum_{j=1}^{i-1} b_{i j M}^{-} \underline{N}_{j}-\sum_{j=1}^{n} b_{i j M}^{+} \bar{N}_{j}\right)\right\} \\
\underline{N}_{i}= & \left(c_{i L}-\sum_{j=1}^{i-1} b_{i j M}^{-} \underline{N}_{j}-\sum_{j \neq i} b_{i j M}^{+} \bar{N}_{j}\right) / \tilde{a}_{i M}, \\
\underline{N}_{i}=\min \left(\underline{\tilde{N}}_{i}, \bar{N}_{i} \exp \left\{\left(c_{i L}-\sum_{j=1}^{i-1} b_{i j M}^{-} \underline{N}_{j}-\sum_{j \neq 1} b_{i j M}^{+} \bar{N}_{j}\right)-\tilde{a}_{i M} \bar{N}_{i}\right\}\right) & >0,1 \leq i \leq n
\end{aligned}\right.
$$

Then, the system (1.1)-(1.2) is permanent.

Moreover, if

$$
\tilde{A}_{L}-\left(B_{M}^{+}-B_{L}^{-}\right) \text {is an } M \text {-matrix, }
$$

then for any two solutions $\left\{M_{i}(p)\right\}_{p=0}^{\infty}$ and $\left\{N_{i}(p)\right\}_{p=0}^{\infty}, 1 \leq i \leq n$ of the system (1.1)-(1.2), it holds

$$
\lim _{p \rightarrow \infty}\left(M_{i}(p)-N_{i}(p)\right)=0, \quad 1 \leq i \leq n .
$$

The organization of this paper is as follows. In Section 2, for the permanence of the system (1.1)-(1.2), in addition to upper bounds $\bar{N}_{i}$ of $\limsup \sup _{p \rightarrow \infty} N_{i}(p), 1 \leq i \leq n$ of Lemma 2.2 in Muroya [13], we obtain new lower bounds $\underline{N}_{i}>0$ of $\liminf \inf _{p \rightarrow \infty} N_{i}(p), 1 \leq i \leq n$ (see Theorem 2.1). For the global asymptotic stability of the system (1.1)-(1.2), applying the recent results in Muroya [13], we establish Theorem 1.1. In particular, for the special case of $n=1$, we find an improved result than those of Muroya [9] and [10] (cf. So and Yu [17]).

\section{Conditions of permanence and global asymptotic stability}

Using the techniques of Muroya [13], we consider the permanence and the global asymptotic stability of the discrete system (1.1)-(1.2) of nonautonomous Lotka-Volterra type.

Lemma 2.1 For the system (1.1)-(1.2), 


$$
\begin{array}{r}
N_{i}(p)=N_{i}(0) \exp \left(\sum_{q=0}^{p-1}\left\{c_{i}(q)-a_{i}(q) N_{i}(q)-\sum_{j=1}^{n} \sum_{l=0}^{m} a_{i j}^{l}(q) N_{j}\left(q-k_{l}\right)\right\}\right) \\
p \geq 1,1 \leq i \leq n, \quad
\end{array}
$$

and every solutions $\left\{N_{i}(p)\right\}_{p=0}^{\infty}, 1 \leq i \leq n$ exist and remain positive for all $p=0,1,2, \ldots$.

Proof. By (1.1), we obtain (2.1), from which we get the conclusion.

Remark 2.1 Consider the following differential equations with piecewise constant delays:

$$
\left\{\begin{array}{c}
\frac{d x_{i}(t)}{d t}=x_{i}(t)\left\{c_{i}([t])-a_{i}([t]) x_{i}([t])-\sum_{j=1}^{n} \sum_{l=0}^{m} a_{i j}^{l}([t]) x_{j}\left(\left[t-k_{l}\right]\right)\right\} \\
t \geq 0,1 \leq i \leq n \\
x_{i}(p)=\phi_{i}(p) \geq 0, \text { for } p \leq 0, \text { and } \phi_{i}(0)>0,1 \leq i \leq n
\end{array}\right.
$$

where $[t]$ denotes the maximal integer less than or equal to $t$ and

$$
\phi_{i}(p)=N_{i p}, \text { for } p \leq 0 .
$$

Then, we easily see that for any $p<t<p+1$, for $p \geq 0$,

$$
\begin{aligned}
& \frac{d}{d t}\left\{\frac{1}{x_{i}(t)} \exp \left(\int_{p}^{t}\left\{c_{i}(p)-a_{i}(p) x_{i}(p)-\sum_{j=1}^{n} \sum_{l=0}^{m} a_{i j}^{l}(p) x_{j}\left(p-k_{l}\right)\right\} d s\right)\right\} \\
& =0 .
\end{aligned}
$$

Thus, integrating both sides with respect to $t$ on $[p, p+1]$, we obtain (1.1) and $N_{i}(p)=x_{i}(p)$, for $p=0,1, \ldots$

For the permanence of the system (1.1)-(1.2), in addition to upper bounds $\bar{N}_{i}$ of $\lim \sup _{p \rightarrow \infty} N_{i}(p), 1 \leq i \leq n$ of Lemma 2.2 in Muroya [13], we obtain new lower bounds $\underline{N}_{i}>0$ of $\liminf _{p \rightarrow \infty} N_{i}(p), 1 \leq i \leq n$.

Theorem 2.1 Under the condition

$$
\left(A_{L}+B_{L}^{-}\right)^{-1} c_{M}>0
$$

for any solutions $N_{i}(p), 1 \leq i \leq n$ of the system (1.1)-(1.2), it holds that

$$
\limsup _{p \rightarrow \infty} N_{i}(p) \leq \bar{N}_{i}, \quad 1 \leq i \leq n
$$


where $\bar{N}_{i}, 1 \leq i \leq n$ are defined by $(1.6)$.

Moreover, if (1.8) holds, then

$$
\liminf _{p \rightarrow \infty} N_{i}(p) \geq \underline{N}_{i}, \quad 1 \leq i \leq n,
$$

where $\underline{N}_{i}, 1 \leq i \leq n$ are defined by (1.9).

Proof. Since $A_{L}+B_{L}^{-}$is an $M$-matrix, it is well known that there is a diagonal matrix $\underline{D}=\operatorname{diag}\left(\underline{d}_{1}, \underline{d}_{2}, \ldots, \underline{d}_{n}\right)$ such that $\underline{d}_{i}>0,1 \leq i \leq n$ and $\left(A_{L}+B_{L}^{-}\right) \underline{D}$ is a diagonally dominant matrix.

Thus, we may assume, without loss of generality, that $A_{L}+B_{L}^{-}$is diagonally dominant, that is, $a_{i L}>0$ and $a_{i L}+\sum_{j=1}^{i-1} b_{i j L}^{-}>0,1 \leq i \leq n$.

Then, (2.5) follows from the proof of Lemma 2.2 in Muroya [13]. that

Hence, for any $\epsilon>0$, there exists a constant $\bar{p}_{n} \geq \max _{0 \leq l \leq m} k_{l}$ such

$$
N_{i}(p) \leq \bar{N}_{i}+\epsilon, \quad p \geq \bar{p}_{n}, \quad 1 \leq i \leq n .
$$

Moreover, assume (1.8) and for some $1 \leq i \leq n$, suppose that for any sufficiently small fixed constant $\epsilon>0$, there exists a constant $\underline{p}_{i} \geq \bar{p}_{n}$ such that

$$
\liminf _{p \rightarrow \infty} N_{j}(p) \geq \underline{N}_{j}-\epsilon>0, \quad 1 \leq j \leq i-1 .
$$

If $N_{i}(p)<\underline{\tilde{N}}_{i}-\left\{\left(-\sum_{j=1}^{i-1} b_{i j M}^{-}+\sum_{j \neq i} b_{i j M}^{+}\right) / \tilde{a}_{i M}\right\} \in$ for some $p \geq \bar{p}_{n}$, then by (1.1) and the definition of $\tilde{N}_{i}$,

$$
\begin{aligned}
N_{i}(p+1) \geq N_{i}(p) \exp \{ & c_{i L}-\sum_{j=1}^{i-1} b_{i j M}^{-}\left(\underline{N}_{j}-\epsilon\right) \\
& \left.-\sum_{j \neq i} b_{i j M}^{+}\left(\bar{N}_{j}+\epsilon\right)-\tilde{a}_{i M} N_{i}(p)\right\}>N_{i}(p) .
\end{aligned}
$$

For the case that $N_{i}(p)$ is eventually increasing and bounded upper by $\underline{\hat{N}}_{i}-$ $\left\{\left(-\sum_{j=1}^{i-1} b_{i j M}^{-}+\sum_{j \neq i} b_{i j M}^{+}\right) / \tilde{a}_{i M}\right\} \epsilon$, we apply the similar discussions to the case $i=1$ in the proof of Lemma 2.2 in Muroya [13], and we get

$$
\liminf _{p \rightarrow \infty} N_{i}(p)=\underline{\tilde{N}}_{i}-\left\{\left(-\sum_{j=1}^{i-1} b_{i j M}^{-}+\sum_{j \neq i} b_{i j M}^{+}\right) / \tilde{a}_{i M}\right\} \epsilon .
$$


On the other hand, if $N_{i}(p) \geq \tilde{N}_{i}-\left\{\left(-\sum_{j=1}^{i-1} b_{i j M}^{-}+\sum_{j \neq i} b_{i j M}^{+}\right) / \tilde{a}_{i M}\right\} \in$ for some $p \geq \bar{p}_{n}$ then by (1.1)

$$
\begin{aligned}
N_{i}(p+1) \geq N_{i}(p) & \exp \left\{\left(c_{i L}-\sum_{j=1}^{i-1} b_{i j M}^{-} \underline{N}_{j}-\sum_{j \neq i} b_{i j M}^{+} \bar{N}_{j}\right)-\tilde{a}_{i M} N_{i}(p)\right\} \\
& \times \exp \left\{-\left(-\sum_{j=1}^{i-1} b_{i j M}^{-}+\sum_{j \neq i} b_{i j M}^{+}\right) \epsilon\right\} .
\end{aligned}
$$

Since for $a>0, \min _{\underline{x} \leq x \leq \bar{x}} x e^{-a x}=\min \left(\underline{x} e^{-a \underline{x}}, \bar{x} e^{-a \bar{x}}\right)$, we get

$$
\begin{array}{r}
\liminf _{p \rightarrow \infty} N_{i}(p) \geq \min \left(\underline{\tilde{N}}_{i} \exp \{-\right. \\
\left.\left(-\sum_{j=1}^{i-1} b_{i j M}^{-}+\sum_{j \neq i} b_{i j M}^{+}\right) \epsilon\right\}-M_{i}^{\epsilon}, \\
\left.\left(\bar{N}_{i}+\epsilon\right) \exp \left\{\underline{c}_{i L}-\tilde{a}_{i M}\left(\bar{N}_{i}+\epsilon\right)\right\}\right),
\end{array}
$$

where

$$
\left\{\begin{aligned}
M_{i}^{\epsilon}=\{(- & \left.\left.\sum_{j=1}^{i-1} b_{i j M}^{-}+\sum_{j \neq i} b_{i j M}^{+}\right) / \tilde{a}_{i M}\right\} \epsilon \\
& \times \exp \left\{-\left(-\sum_{j=1}^{i-1} b_{i j M}^{-}+\sum_{j \neq i} b_{i j M}^{+}\right) \epsilon\right\}, \\
\underline{c}_{i L}=c_{i L}- & \sum_{j=1}^{i-1} b_{i j M}^{-} \underline{N}_{j}-\sum_{j \neq i} b_{i j M}^{+} \bar{N}_{j}, \quad 1 \leq i \leq n .
\end{aligned}\right.
$$

Since $\epsilon>0$ is any sufficiently small positive constant, we have that

$$
\liminf _{p \rightarrow \infty} N_{i}(p) \geq \underline{N}_{i}, \quad 1 \leq i \leq n
$$

Hence, by inductions, we complete the proof.

Remark 2.2 Note that the condition (1.8) is a sufficient condition for the permanence of the system (1.1)-(1.2) (cf. (1.22) and (1.24) in Muroya [13]).

Recently, Muroya [13] has established a result which is an improved version of known conditions for the global asymptotic stability in discrete system (1.1)-(1.2) (cf. Gopalsamy [4]-[6], Tineo and Alvalez [18], Redheffer [14], Ahmad and Lazer [1]-[2] and Wang et al. [20]). 
Lemma 2.2 For the system (1.1)-(1.2), assume the conditions (1.5) and (1.8), and suppose that there exist positive constants $\alpha_{1}, \alpha_{2}, \ldots, \alpha_{n}, \eta>0$ and a positive integer $p_{0}$ such that for $p \geq p_{0}$,

$$
\alpha_{i} \tilde{a}_{i}(p)-\sum_{j \neq i} \alpha_{j}\left|a_{j i}^{0}(p)\right|-\sum_{j=1}^{n} \alpha_{j} \sum_{l=1}^{m}\left|a_{j i}^{l}\left(p+k_{l}\right)\right| \geq \eta, \quad 1 \leq i \leq n
$$

where

$$
\tilde{a}_{i}(p)=\min \left(a_{i}(p), \frac{2}{\bar{N}_{i}}-a_{i}(p)\right), \quad 1 \leq i \leq n .
$$

Then any two solutions $\left\{M_{i}(p)\right\}_{p=0}^{\infty},\left\{N_{i}(p)\right\}_{p=0}^{\infty}, 1 \leq i \leq n$ of the system (1.1)-(1.2), satisfy the condition

$$
\lim _{p \rightarrow \infty}\left(M_{i}(p)-N_{i}(p)\right)=0, \quad 1 \leq i \leq n .
$$

Proof. The proof of this theorem follows from Theorem 2.1 and the proof of Lemma 2.8 in Muroya [13].

By Lemma 2.2, in order to prove Theorem 1.1, it is sufficient to show that the condition (1.10) implies the existence of $\alpha_{i}>0,1 \leq i \leq n$ such that (2.7) holds. We can see that (1.10) implies stronger inequalities in (2.10) (cf. Ahmad and Lazer [2, Lemma 3.2]).

Lemma 2.3 (See Berman and Plemmons [3, p. 137]) (1.10) holds, if and only if there exist constants $\alpha_{i}>0,1 \leq i \leq n$ such that for $1 \leq i \leq n$,

$$
\begin{array}{r}
\alpha_{i} \tilde{a}_{i L}-\sum_{j \neq i} \alpha_{j}\left(a_{j i M}^{0+}-a_{j i L}^{0-}\right)-\sum_{j=1}^{n} \alpha_{j} \sum_{l=1}^{m}\left(a_{j i M}^{l+}-a_{j i L}^{l-}\right)>0, \\
1 \leq i \leq n .
\end{array}
$$

From Theorem 1.1, we easily obtain the following corollary.

Corollary 2.1 (Cf. Theorem 1 in Wang et al. [20]) For the system (1.1)(1.2) and (1.6), if

$$
\left\{\begin{array}{l}
\tilde{c}_{i M} \leq a_{i L} / a_{i M} \leq 1 \text { and } \bar{N}_{i}=\tilde{c}_{i M} / a_{i L}, \quad 1 \leq i \leq n \\
\left(A_{L}+B_{L}^{-}\right)^{-1} c_{M}>\mathbf{0}, c_{L}>\left(B_{M}^{+}-D_{M}^{+}\right)\left(A_{L}+B_{L}^{-}\right)^{-1} c_{M} \\
\text { and } A_{L}-\left(B_{M}^{+}-B_{L}^{-}\right) \text {is an } M \text {-matrix }
\end{array}\right.
$$


then for any two solutions $\left\{M_{i}(p)\right\}_{p=1}^{\infty}$ and $\left\{N_{i}(p)\right\}_{p=1}^{\infty}, 1 \leq i \leq n$ of the system (1.1)-(1.2), (1.11) holds.

Proof. By (1.6) and (2.11), we have $\bar{N}_{i}=\tilde{c}_{i M} / a_{i L} \leq 1 / a_{i M}, 2 / \bar{N}_{i}-a_{i M} \geq$ $1 / \bar{N}_{i}=a_{i L} / \tilde{c}_{i M} \geq a_{i L}, 1 \leq i \leq n$ and $\bar{N}=\left(A_{L}+B_{L}^{-}\right)^{-1} c_{M}$. Then, in this case, (2.11) implies (1.5), (1.8) and (1.10). Therefore, by Theorem 1.1, we obtain (1.7) and (1.11).

Remark 2.3 In Theorem 1 in Wang et al. [20], it is assumed the condition that the system (1.1)-(1.2) is strongly persistent, that is, $\lim \inf _{p \rightarrow \infty} N_{i}(p)>$ $0,1 \leq i \leq n$. For the competitive system with $n=1$ and 2 , Wang et al. [20] has given sufficient conditions similar to (2.11), which are improved and extended by Theorem 1.1 to $n \geq 1$ (see also Corollary 2.2 and Remark 2.4).

Consider the following differential equation with piecewise constant arguments:

$$
\left\{\begin{array}{r}
\frac{d N(t)}{d t}=N(t) r(t)\left\{1-b_{0} N(p)-\sum_{i=1}^{m} b_{i} N(p-i)\right\} \\
p \leq t<p+1, p=0,1,2, \ldots, \\
N(0)=N_{0}>0, \text { and } N(-j)=N_{-j} \geq 0,1 \leq j \leq m,
\end{array}\right.
$$

where each $r(t)$ is a nonnegative continuous function on $[0, \infty), r(t) \not \equiv 0$,

$$
b_{0}>0, \text { and } b_{i} \geq 0,1 \leq i \leq m .
$$

Then, similar to the proof in Remark 2.1, we have that

$$
\left\{\begin{array}{r}
N(p+1)=N(p) \exp \left\{r_{p}\left(1-b_{0} N(p)-\sum_{i=1}^{m} b_{i} N(p-i)\right)\right\} \\
p=0,1,2, \ldots \\
N(0)=N_{0}>0, \text { and } N(-j)=N_{-j} \geq 0,1 \leq j \leq m
\end{array}\right.
$$

where

$$
r_{p}=\int_{p}^{p+1} r(t) d t, \quad p \geq 0 .
$$

To this system, we apply Theorem 1.1 and get the following corollary. 
Corollary 2.2 For (2.12)-(2.13), assume that

$$
\left\{\begin{array}{l}
b_{0}>\sum_{i=1}^{m} b_{i}, \liminf _{p \rightarrow \infty} r_{p}>0 \text { and } \\
\bar{r}=\sup _{p \geq 0} r_{p}<1+\ln \left\{2 b_{0} /\left(\sum_{i=0}^{m} b_{i}\right)\right\}<1+\ln 2 .
\end{array}\right.
$$

Then, the positive equilibrium $N^{*}=1 /\left(\sum_{i=0}^{m} b_{i}\right)$ of $(2.12)-(2.13)$ is globally asymptotically stable.

Proof. For (2.14), the condition $\liminf _{p \rightarrow \infty} r_{p}>0$ implies (1.8). Now, put

$$
\bar{N}= \begin{cases}\frac{1}{b_{0}}, & \bar{r} \leq 1, \\ \frac{e^{\bar{r}-1}}{\bar{r} b_{0}}, & \bar{r}>1 .\end{cases}
$$

If $\bar{r} \leq 1$, then from the conditions (2.13) and $b_{0}>\sum_{i=1}^{m} b_{i}$, we have $\min \left(\bar{r} b_{0}, \frac{2}{\bar{N}}-\bar{r} b_{0}\right)=\bar{r} b_{0}>\bar{r} \sum_{i=1}^{m} b_{i}$, which implies (1.10) for (2.14).

If $\bar{r}>1$, then by $(2.16), e^{\bar{r}-1}<2 b_{0} /\left(\sum_{i=0}^{m} b_{i}\right)$ and from $\bar{r} \sum_{i=0}^{m} b_{i}<$ $\frac{2 \bar{r} b_{0}}{e^{\bar{r}-1}}=\frac{2}{\bar{N}}$ we have that

$$
\min \left(\bar{r} b_{0}, \frac{2}{\bar{N}}-\bar{r} b_{0}\right)>\bar{r} \sum_{i=1}^{m} b_{i}
$$

from which we get (1.10) for (2.14). Thus, by Theorem 1.1, we obtain the conclusion of this corollary.

Remark 2.4 The condition (2.16) in Corollary 2.2 improve the sufficient conditions of (2.9) that $b_{0}>\sum_{i=1}^{m} b_{i}$ and $r_{p} \leq 1$ in Muroya [9] which has the "contractivity condition". Similarly, Theorem 3 in Wang et al. [20] is also improved by Theorem 1.1.

So and $\mathrm{Yu}$ [17] have established sufficient conditions that $\limsup \sup _{p \rightarrow \infty} r_{p}>0$ and $r_{p} \leq \frac{3}{2(m+1)}$ for (2.12)-(2.13), and Muroya [10] offer the sufficient condition $r_{p} \leq \hat{r}(\tilde{\alpha})=f(0 ; \hat{r}(\tilde{\alpha}))<\sup _{t<1} f(t ; \hat{r}(\tilde{\alpha}))=$ $2 b_{0} /\left(\sum_{i=0}^{m} b_{i}\right), \tilde{\alpha}=1-\left(\sum_{i=0}^{m} b_{i}\right) / b_{0}<0$, where $\hat{r}(\tilde{\alpha}), f(t ; r)$ are defined in [10]. Thus, the condition (2.16) is other type condition than those of So and $\mathrm{Yu}$ [17] and Muroya [10]. From these results, we have a conjecture that there is a larger region of sufficient conditions which contains all the above conditions. To solve this conjecture is our future work. 
Acknowledgement The author grateful to the referee for his careful reading and many constructive comments.

\section{References}

[1] Ahmad S. and Lazer A.C., Necessary and sufficient average growth in a LotkaVolterra system. Nonlinear Analysis 34 (1998), 191-228.

[2] Ahmad S. and Lazer A.C., Average conditions for global asymptotic stability in a nonautonomous Lotka-Volterra system. Nonlinear Analysis 40 (2000), 37-49.

[3] Berman A. and Plemmons R.J., Nonnegative Matrices in Mathematical Sciences. Academic Press, New York (1979).

[4] Gopalsamy K., Global asymptotic stability in Volterra's population systems. J. Math. Biol. 19 (1984), 157-168.

[5] Gopalsamy K., Global asymptotic stability in a periodic Lotka-Volterra system. J. Austral. Math. Soc. Ser B 27 (1985), 66-72.

[6] Gopalsamy K., Global asymptotic stability in an almost-periodicLotka-Volterra system. J. Austral. Math. Soc. Ser B 27 (1986) 346-360.

[7] Hofbaur J., Hutson V. and Jansen W., Coexistence for systems governed by difference equations of Lotka-Volterra type. J. Math. Biol. 25 (1987), 553-570.

[8] Lu Z. and Wang W., Permanence and global attractivity for Lotka-Volterra difference systems. J. Math. Biol. 39 (1999), 269-282.

[9] Muroya Y., A sufficient condition on global stability in a logistic equation with piecewise constant arguments. Hokkaido Math. J. 32 No.1 (2003), 75-83.

[10] Muroya Y., Persistence, contractivity and global stability in logistic equations with piecewise constant delays. J. Math. Anal. Appl. 270 (2002), 602-635.

[11] Muroya Y., Uniform persistence for Lotka-Volterra type delay differential systems. Nonlinear Analysis RWA 4 (2003), 689-710.

[12] Muroya Y., Persistence and global stability for nonautonomous Lotka-Volterra delay differential systems. Communications on Applied Nonlinear Analysis 9 (2002), 3145.

[13] Muroya Y., Persistence and global stability for discrete models of nonautonomous Lotka-Volterra type. J. Math. Anal. Appl. 273 (2002), 492-511.

[14] Redheffer R., Nonautonomous Lotka-Volterra system I. J. Differential Equations 127 (1996), 519-540.

[15] Saito Y., Hara T. and Ma W., Harmless delays for permanence and impersistence of Lotka-Volterra discrete predator-prey sysrem. Nonlinear Analysis 50 (2002), 703715.

[16] "Saito Y., Ma W. and Hara T., A necessary and sufficient condition for permanence of a Lotka-Volterra discrete system with delays. J. Math. Anal. Appl. 256 (2001), $162-174$.

[17] So J.W.-H. and Yu J.S., Global stability in a logistic equation with piecewise constant arguments. Hokkaido Math. J. 24 (1995), 269-286. 
[18] Tineo A. and Alvarez C., A different consideration about the globally asymptotically stable solution of the periodic n-competing species problem. J. Math. Anal. Appl. 159 (1991), 44-60.

[19] Wang W. and Lu Z., Global stability of discrete models of Lotka-Volterra type. Nonlinear Analysis 35 (1999), 1019-1030.

[20] Wang W., Mulone G., Salemi F. and Salone V., Global stability of discrete population models with time delays and fluctuating environment. J. Math. Anal. Appl. 264 (2001), 147-169.

Department of Mathematical Sciences Waseda University 3-4-1 Ohkubo Shinjuku-ku Tokyo 169-8555, Japan

E-mail: ymuroya@waseda.jp 